Revue d'histoire de l'Amérique française

MAU REVUE D.HISTOIRE DE L'AMÉRIQUE FRANÇAISE

\title{
La construction navale à Québec, 1760-1825 : sources inexplorées et nouvelles perspectives de recherches
}

\section{Pierre Dufour}

Volume 35, numéro 2, septembre 1981

URI : https://id.erudit.org/iderudit/303952ar

DOI : https://doi.org/10.7202/303952ar

Aller au sommaire du numéro

\section{Éditeur(s)}

Institut d'histoire de l'Amérique française

\section{ISSN}

0035-2357 (imprimé)

1492-1383 (numérique)

Découvrir la revue

\section{Citer cet article}

Dufour, P. (1981). La construction navale à Québec, 1760-1825 : sources inexplorées et nouvelles perspectives de recherches. Revue d'histoire de l'Amérique française, 35(2), 231-251. https://doi.org/10.7202/303952ar d'utilisation que vous pouvez consulter en ligne. 


\title{
LA CONSTRUCTION NAVALE À QUÉBEC, 1760-1825: SOURCES INEXPLORÉES ET NOUVELLES PERSPECTIVES DE RECHERCHES
}

\author{
PIERRE DUFOUR \\ historien \\ Parcs Canada, Québec
}

Le présent article expose quelques résultats obtenus au terme de la première phase d'une recherche portant sur l'histoire de la construction navale à Québec aux XVIII ${ }^{\mathrm{e}}$ et $\mathrm{XIX}^{\mathrm{e}}$ siècles ${ }^{*}$. En premier lieu, nous résumons brièvement les connaissances historiographiques concernant cette activité entre 1760 et 1900, puis nous démontrons comment les sources utilisées jusqu'ici par les historiens pour mesurer l'ampleur du phénomène les ont amenés à le surévaluer. Ensuite, nous donnons un aperçu de la construction navale qui se pratiquait dans l'ensemble de la vallée du SaintLaurent entre 1760 et 1825 , puis nous tentons de dégager l'importance relative des chantiers maritimes de la ville de Québec au cours de la même période. Notre exposé repose uniquement sur une nouvelle source - nouvelle au sens où elle n'a pas encore été exploitée systématiquement: le certificat d'enregistrement de navires, qui mérite d'être d'abord présenté sommairement.

\section{Le certificat d'enregistrement de navires}

En 1786, le Parlement britannique entérine la loi 26 Geo. III c. 60 pour encourager le commerce et la navigation'. Cette loi prévoit, entre autres, que seuls les navires britanniques pourront jouir désormais de certains droits et privilèges sur le plan du commerce maritime. En vertu de cette loi, ne sont considérés comme navires britanniques que ceux qui sont construits en Angleterre ou dans ses colonies et qui sont possédés et affrétés par des sujets britanniques. Afin d'assurer sa mise en application, la loi stipule aussi que

* Cette étude sur la construction navale s'inscrit dans le cadre plus général d'un programme de recherches sur l'histoire du port de Québec; l'auteur travaille, à titre d'historien, pour Parcs Canada, Québec. Le texte présenté ici a pu être rédigé grâce à l'étroite collaboration de M. Marc Ouellet.

1 Les modalités d'application de cette loi sont expliquées en détail dans David Steele, The Ship-Master's Manual and Owner's Manual $\left(12^{\mathrm{e}}\right.$ éd., Londres, Steele \& Co., 1810), 400-415; nous résumons ici les principaux points concernant plus spécifiquement le certificat d'enregistrement comme tel. 
tout propriétaire d'un navire comportant un pont ou jaugeant plus de 15 tonneaux doit se prémunir, auprès d'un officier des douanes, d'un certificat d'enregistrement.

Quiconque désire obtenir un tel certificat doit remettre à l'officier autorisé un document produit par le constructeur du navire, indiquant où et quand celui-ci a été construit, spécifiant sa jauge et identifiant son ou ses propriétaires. L'officier de la douane transpose ces renseignements sur le certificat d'enregistrement, de même que plusieurs autres données ayant trait aux caractéristiques techniques du navire. Chaque certificat doit être reproduit en trois exemplaires: le premier pour être remis au propriétaire du bâtiment ou à son capitaine, le second pour être inséré dans un registre conservé au port où l'enregistrement est effectué, et le troisième pour être expédié à Londres.

Les registres de certificats d'enregistrement de navires émis au port de Québec remontent à l'année 1787 et ils sont conservés aux Archives publiques du Canada, dans le fonds "Direction de la Marine, 1762-1966» (RG 42). Ces documents s'avèrent fort précieux, voire essentiels, pour toute étude sur la construction navale à Québec ${ }^{2}$. Leur exploitation ne permet sans doute pas de faire toute la lumière sur les pourquoi et les comment de cette activité, mais elle nous a conduit à remettre en question certaines idées reçues de même qu'à identifier quelques phénomènes qui semblent avoir échappé aux historiens jusqu'à présent.

\section{Survol historiographique}

Il n'existe pratiquement pas d'études portant sur la construction navale à Québec entre 1760 et 1900. À part Albert Faucher qui a tenté de dégager les causes du déclin de cette industrie au cours de la seconde moitié du XIX siècle, seulement quelques auteurs ont rédigé de courts articles à saveur essentiellement anecdotique sur le sujet ${ }^{3}$. Par contre, les historiens qui ont étudié l'évolution de la ville de Québec tout comme d'autres qui se sont penchés sur l'histoire économique du Canada, l'ont abordée presque invariablement. Ils sont nombreux à avoir signalé l'importance de la construction navale au sein de l'économie canadienne durant une bonne partie du $\mathrm{XIX}^{\mathrm{e}}$ siècle, ou à avoir souligné sa primauté

2 Sur le degré de fiabilité du certificat d'enregistrement de navires comme source pour l'histoire de la construction navale, voir l'article de Richard Rice, «Measuring British Dominance of Shipbuilding in the 'Maritimes', 1787-1890», paru dans K. Mathews et G. Paning, Ships and Shipbuilding in the North Atlantic Region; Proceedings of the Conference on the Atlantic Canada Shipping Project, March 31 - April 2, 1977 (St. John's, Memorial University of Newfoundland, 1978), 111-155. 1970), 227-254.

Albert Faucher, Histoire économique et unité canadienne (Montréal, Fides, 
comme pôle de croissance pour le développement de la ville de Québec.

Ainsi, Le Vasseur affirme: «La construction navale fut, pendant plus de deux cents ans, après l'agriculture, la première et la plus importante industrie du Canada, voire même de l'Amérique du Nord.» ${ }^{4}$ Un autre auteur abonde dans le même sens pour hisser cette industrie au rang de "la plus grande source de richesse en notre pays» ${ }^{5}$. Aux yeux d'un historien de la ville de Québec, «deux industries ont successivement assuré le pain quotidien» à sa population: la construction navale et la fabrication de la chaussure ${ }^{6}$. Fernand Ouellet considère aussi la construction navale comme «l'industrie essentielle de la région de Québec» durant la première moitié du XIX ${ }^{\mathrm{e}}$ siècle, opinion partagée par Faucher: «La construction de navires en bois fut une des réalisations marquantes du Québec au XIX ${ }^{\mathrm{e}}$ siècle [...] c'était l'industrie principale de la ville de Québec.»> Bref, les jugements de cette nature sont fréquents.

Cependant, pour qui cherche à estimer de façon plus précise l'importance relative de la construction navale à Québec en recourant à l'historiographie, de sérieuses difficultés surgissent. Les seules données quantitatives que nous avons pu extraire des travaux des historiens ne portent que sur deux ordres de phénomènes: le nombre de personnes employées à la construction navale à divers moments et la quantité de bâtiments sortis annuellement des chantiers maritimes de Québec.

Toutefois, les éléments quantitatifs sur la main-d'oeuvre s'avèrent fort rares. Nous savons que de novembre 1821 à 1822 , 700 immigrants ont pu travailler temporairement dans les chantiers

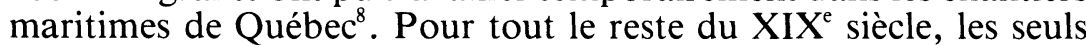
chiffres qui ont pu être colligés apparaissent au tableau 1 .

Il n'y a donc pas là matière suffisante pour se former une idée juste de l'importance réelle de la construction navale. Et ce, d'autant plus que les auteurs qui fournissent ces chiffres ne les confrontent pas avec des données comparables comme, par exemple, la

4 N. Le Vasseur, «La construction des navires à Québec», Bulletin de la Société de Géographie de Québec, 2, 4 (1917): 187.

5 Marius Barbeau, «Constructeurs de navires», Le Canada français (Québec), 28, 8-9 (avril-mai 1940): 907. 1948), 132.

6 Albert Jobin, La petite histoire de Québec (Québec, Institut Saint-Jean-Bosco,

Fernand Ouellet, Histoire de la Chambre de Commerce de Québec (Québec, Université Laval, 1959), 57; Faucher, op. cit., 227.

8 Fernand Ouellet, Le Bas-Canada, 1791-1840; changements structuraux et crise (Ottawa, Éditions de l'Université d'Ottawa, 1976), 220. 


\section{TABLEAU 1}

Main-d'oeuvre employée dans les chantiers maritimes de Québec au XIX ${ }^{\mathrm{e}}$ siècle

\begin{tabular}{|c|c|}
\hline Années & Nombre d'ouvriers \\
\hline 1825 & 33559 \\
\hline 1831 & $1155 \quad 10$ \\
\hline 1847 & $4600 \quad 11$ \\
\hline 1860 & 220012 \\
\hline 1867 & $1400 \quad 13$ \\
\hline 1873 & $800 \quad 14$ \\
\hline
\end{tabular}

population ouvrière totale aux mêmes dates ou encore la proportion de personnes employées dans d'autres secteurs d'activité.

Les historiens tablent encore plus volontiers sur le critère de la production que sur celui de la main-d'oeuvre pour évaluer l'importance de cette industrie. C'est à l'aulne du nombre de bâtiments lancés annuellement et de leur tonnage global qu'ils mesurent le plus souvent l'évolution de la construction navale à Québec. En général, ils puisent leurs données quantitatives dans un répertoire des navires construits à Québec au XIX ${ }^{\mathrm{e}}$ siècle, publié en 1897 par un ancien constructeur de navires à la retraite, Narcisse Rosa.

Rosa présente lui-même son ouvrage comme étant «un tableau complet du nombre et de la classe de navires construits $\grave{a}$ Québec depuis 1797 à $1896 »^{15}$. Il y recense, année après année et unité par unité, tous les vaisseaux construits à Québec, en fournissant pour chacun: le nom du navire, les noms de son constructeur et de son premier capitaine, ainsi que quelques caractéristiques techniques du bâtiment (dimensions, tonnage et classe). Rosa conclut cette énumération de plus d'une centaine de pages par le paragraphe suivant: «D'après ce tableau, qui est aussi exact qu'il peut l'être, il y a eu, de 1797 à 1896, 2542 navires de construits à Québec et ses environs, représentant une capacité de 1377099 tonneaux.»1 ${ }^{16}$

La plupart des historiens qui ont écrit sur le sujet par la suite se sont évidemment fiés à ce laborieux travail pour évaluer la pro-

9 Fernand Ouellet, Histoire économique et sociale du Québec, 1760-1850; structures et conjonctures (Montréal, Fides, 1966), 423.

10 Ouellet, Histoire économique, 403.

Ibid., 503.

Jobin, op cit., 147.

Jean Hamelin et Yves Roby, Histoire économique du Québec, 1851-1896 (Montréal, Fides, 1971), 275-276.

14 Jobin, op. cit., 147.

15 Narcisse Rosa, La construction des navires à Québec et ses environs; grèves et naufrages (Québec, Léger Brousseau, 1897), 21; le souligné est de nous.

16 Ibid., 158; c'est nous qui soulignons à nouveau. 
duction des chantiers maritimes de Québec au XIX ${ }^{\mathrm{e}}$ siècle ${ }^{17}$. Mais ce faisant, ils ont été induits en erreur, car la liste des navires produite par Rosa ne correspond pas, et de loin, à la réalité. Il est cependant possible de rectifier ces données en utilisant les registres de certificats d'enregistrement de navires émis au port de Québec à compter de 1787.

Par exemple, pour l'année 1815, Rosa énumère 17 navires construits à Québec alors que seulement quatre d'entre eux l'ont effectivement été, les autres étant sortis de chantiers maritimes établis ailleurs dans la vallée du Saint-Laurent, et même en Angleterre et aux États-Unis. De plus, neuf des 17 navires mentionnés par Rosa ont été lancés non pas en 1815, mais entre 1804 et 1814 . Enfin, les 13 personnes présentées par Rosa comme étant les constructeurs des mêmes 17 bâtiments, sont en réalité leurs propriétaires: si trois d'entre eux s'identifient comme constructeurs de navires, les dix autres sont marchands, charpentiers ou navigateurs. Sans compter ces nombreuses erreurs d'identité de personnes, le répertoire de Rosa surévalue donc grandement la production des chantiers maritimes de Québec. Et l'année 1815 ne constitue pas une exception. Une analyse rapide portant sur les vingt premières années de son recensement permet de le constater.

De 1797 à 1817 inclusivement, Rosa répertorie 296 vaisseaux construits à Québec et dans les environs, tandis que les certificats d'enregistrement attestent que seulement 156 d'entre eux y ont réellement été lancés ${ }^{18}$. Les 140 autres bâtiments? Leurs lieux d'origine varient considérablement. Des chantiers implantés bien en amont de la ville de Québec, tels Bécancour, Trois-Rivières, Sorel, Terrebonne et Montréal, en ont construit 57, alors que 26 autres ont été mis en chantier dans des localités à l'est de Québec, depuis Baie Saint-Paul jusqu'à la Baie des Chaleurs inclusivement. Quant aux 57 bâtiments supplémentaires énumérés par Rosa, ils sont bien

17 Bon nombre d'auteurs ont repris intégralement la conclusion formulée par Rosa: George Gale, Historic Tales of Old Quebec (Quebec, Telegraph Printing Co., 1923), 140; Barbeau, op. cit., 907; Damase Potvin, Le Saint-Laurent et ses îles; histoire, légendes, anecdotes, descriptions, topographie (Québec, Garneau, 1945), 13; Ouellet, Chambre de Commerce, 57; Ivan S. Brookes, The Lower St. Lawrence; a Pictorial History of Shipping and Industrial Development (Cleveland, Freshwater Press, 1974), 9; Pour sa part, Jacques Mathieu, dans son étude sur La construction navale royale à Québec, 1739-1759 (Québec, Société historique de Québec, 1971, 53), a utilisé l'ouvrage de Rosa pour compiler le nombre de bâtiments d'une jauge supérieure à 100 tonneaux, lancés à Québec de 1797 à 1835 . Enfin, Faucher s'est lui aussi servi des données de Rosa pour tracer une courbe de l'évolution de la production des chantiers maritimes de Québec au XIX ${ }^{\mathrm{e}}$ siècle (Faucher, op. cit., 229).

18 Les chantiers maritimes de la ville de Québec ont produit 130 de ces bâtiments, les autres provenant de localités situées dans un rayon d'une cinquantaine de km: AngeGardien, île d'Orléans, Berthier-en-Bas, Etchemin, New Liverpool, Cap-Santé, Portneuf et Deschambault. 
«sortis» des chantiers maritimes de la ville de Québec, mais après y avoir subi des modifications ou des réparations, non pas pour y avoir été construits; parmi ces derniers, plus d'une trentaine étaient d'ailleurs des "prises», c'est-à-dire des voiliers battant pavillon étranger à celui de l'Angleterre, qui avaient été capturés par des navires britanniques puis conduits aux chantiers maritimes de Québec pour y être remis en état ${ }^{19}$.

$\mathrm{Au}$ total, la proportion des bâtiments contenus dans le répertoire de Rosa qui ont réellement été mis en chantier à Québec entre 1797 et 1817, n'est que de 43,91 pour cent. Même en y ajoutant ceux lancés dans les environs de Québec, cette proportion ne dépasse que légèrement la moitié, pour atteindre 52,7 pour cent. Mais en tenant compte des voiliers produits dans les localités sises en amont et en aval de Québec et de sa région immédiate, la proportion grimpe à 80,74 pour cent. En somme, l'ouvrage de Rosa reflète davantage l'importance de la construction navale pour l'ensemble de la vallée du Saint-Laurent que pour la seule ville de Québec. Et pour avoir puisé à cette source, la plupart des historiens ont confondu le nombre de bâtiments construits dans la vallée laurentienne avec celui des bâtiments sortis des chantiers de Québec.

Quelques historiens ont cependant évité ce piège puisqu'ils se sont basés sur une compilation du nombre de bâtiments construits annuellement à Québec, effectuée régulièrement par le Bureau de la Douane. Ces bilans paraissaient souvent dans des journaux de l'époque comme le Quebec Mercury et le Morning Chronicle ainsi que dans diverses publications gouvernementales telles les Journaux de l'Assemblée législative ou certains rapports annuels du Ministère des Travaux publics. Pourtant, les auteurs qui ont recouru à ces statistiques officielles surévaluent la production des chantiers maritimes de Québec et ce, dans une proportion encore plus grande que les utilisateurs du répertoire de Rosa. En effet, selon Le Vasseur, 3627 bâtiments ont été construits à Québec entre 1788 et 1872, tandis que Jobin en dénombre 3872 entre 1787 et 1897, soit un écart de plus d'un millier d'unités par rapport aux chiffres de Rosa ${ }^{20}$. Sans doute ces auteurs ignoraient-ils que l'ex-

19 Les 57 bâtiments qui ont ainsi subi des réparations à Québec provenaient d'Angleterre, d'Écosse, de Terre-Neuve, du Nouveau-Brunswick, des États-Unis et des Antilles.

20 Le Vasseur, op. cit., 200; Jobin, op. cit., 147. Fernand Ouellet s'est lui aussi servi des statistiques du Bureau de la Douane pour tracer un graphique de la production annuelle des chantiers de Québec, exprimée en tonnage global (Ouellet, Histoire économique, 614). Or, son graphique coïncide sensiblement avec celui préparé par Faucher à partir des données de Rosa. Si l'écart s'amoindrit lorsque le tonnage global plutôt que le nombre de bâtiments est choisi pour unité de mesure, c'est que les statistiques émises par le Bureau de la Douane tiennent compte des bateaux à vapeur et des bâtiments d'une jauge inférieure à 100 tonneaux, catégories que Rosa n'a pas inclues dans son répertoire. 
pression «port de Québec» utilisée dans les documents administratifs du XIX ${ }^{\mathrm{e}}$ siècle, recouvre une réalité géographique beaucoup plus vaste que la seule zone des aménagements portuaires de la ville de Québec même, de sorte que les statistiques du Bureau de la Douane fournissent en réalité un bilan de la construction navale pratiquée dans la majorité des chantiers de la vallée laurentienne ${ }^{21}$.

En définitive, l'historiographie a donc transmis à venir jusqu'à présent une image pour le moins fort imprécise de la construction navale à Québec au XIX ${ }^{\mathrm{e}}$ siècle, floue quant à la maind'oeuvre et inexacte quant à la production des chantiers maritimes. En outre, cette image nous laisse nettement l'impression que la construction navale n'a revêtu une importance prépondérante que pour la seule ville de Québec, voire qu'elle y était presque exclusivement pratiquée, impression fausse, imputable au fait que les deux principales sources utilisées par les historiens pour étudier cette industrie, masquent l'existence d'une réalité beaucoup plus complexe et nuancée.

\section{La construction navale dans la vallée du Saint-Laurent, 1760-1825}

Les études historiques ne livrent que de rares indices sur l'existence de chantiers maritimes ailleurs qu'à Québec, entre 1760 et 1825. Résumons brièvement ces quelques éléments de connaissance. De mai 1761 à mai 1763, une douzaine de bâtiments sont lancés dans la région de Trois-Rivières ${ }^{22}$. Dès avant 1805 , un constructeur de navires oeuvre à Montréal où quelques navires sont lancés chaque année à cette date ${ }^{23}$. En 1809, John Molson fait construire au même endroit le troisième bateau à vapeur de l'histoire à assurer une liaison commerciale régulière, ce qui laisse supposer qu'on y construit toujours des bâtiments ${ }^{24}$. A compter de

$21 \quad$ Au début du XIX $\mathrm{X}^{\mathrm{e}}$ siècle, ces compilations effectuées par le Bureau de la Douane englobent même la production de la totalité des chantiers maritimes établis le long du fleuve ou de ses affluents, depuis Montréal jusqu'au golfe inclusivement; plus tard, l'entité administrative désignée par l'expression «port de Québec» varie à quelques reprises, mais elle ne correspond jamais aux aménagements portuaires de Québec même, ces derniers étant plutôt appelés «hâvre de Québec» dans les mêmes documents. L'erreur d'interprétation signalée ci-haut est d'autant plus compréhensible que rien, dans la présentation des statistiques portant sur la construction navale et qui émanent du Bureau de la Douane, ne laisse soupçonner ce fait: dans leur version anglaise, les compilations sont précédées de la mention: «Number of vessels built at the port of Quebec», sans aucune autre précision, tandis que la version française affiche habituellement un titre encore plus trompeur pour omettre le terme «port»: «Nombre des navires construits à Québec».

22 Marcel Trudel, Le Régime militaire dans le Gouvernement de Trois-Rivières, 1760-1764 (Trois-Rivières, Éditions du Bien Public, 1952), 101-102.

23 Gilles Paquet et Jean-Pierre Wallot, «Le Bas-Canada au début du XIX ${ }^{\mathrm{e}}$ siècle: une hypothèse», Revue d'histoire de l'Amérique française, 25, 1 (juin 1971): 50.

24 Alfred Dubuc, «Montréal et les débuts de la navigation à vapeur sur le SaintLaurent», Revue d'histoire économique et sociale, 45 (1967): 110. 
1815, plusieurs voiliers et navires à vapeur sont régulièrement assemblés dans différents chantiers montréalais, situés pour la plupart à Hochelaga, et au début des années 1820, Montréal possède deux fonderies produisant des moteurs pour des navires ${ }^{25}$. À la même époque, Sorel lance aussi des bâtiments pour des clients montréalais ${ }^{26}$. Ce sont là les seuls chantiers maritimes de la vallée laurentienne dont l'existence est actuellement certifiée par des études historiques ${ }^{27}$.

Or, comme nous l'avons déjà laissé entrevoir, les registres de certificats d'enregistrement de navire émis au port de Québec révèlent que la construction navale se pratique en de nombreux endroits de la vallée du Saint-Laurent, depuis les îles-de-laMadeleine et la côte du Labrador à l'est, jusqu'à Montréal, à l'ouest. Entre 1760 et 1800 seulement, pas moins d'une cinquantaine de localités - villages et lieux dits - produisent des bâtiments, et pendant le premier quart du XIX ${ }^{\mathrm{e}}$ siècle, une quarantaine d'autres s'y ajoutent.

La taille des bâtiments mis en chantier au cours de cette période dans l'ensemble $\mathrm{du}$ territoire s'avère relativement modeste: 60 pour cent des voiliers construits jaugent moins de 100 tonneaux, et un autre quart de la production totale est constitué de bâtiments jaugeant entre 100 et 300 tonneaux. De plus, même si une dizaine de classes différentes de voiliers sont construits, trois d'entre elles comptent pour plus des trois-quarts de la production totale $(76,79$ pour cent).

La goélette, ou schooner, compose à elle seule plus de la moitié de tous les bâtiments lancés. Création typiquement nordaméricaine, la goélette est apparue simultanément en NouvelleAngleterre et en Nouvelle-France vers la fin du XVII ${ }^{e}$ siècle $^{28}$. Les goélettes construites dans la vallée du Saint-Laurent entre 1760 et 1825 oscillent entre 30 et 180 tonneaux. Malgré leur taille res-

25 Gerald J. J. Tulchinsky, The River Barons: Montreal Businessmen and the Growth of Industry and Transportation, 1837-1853 (Toronto, University of Toronto Press, 1977), 8, 11 .

26 Ibid., 11.

27 Toutefois, durant la Révolution américaine et la guerre de 1812, d'éphémères chantiers navals ont aussi existé pour des fins militaires à Sorel, Chambly, Saint-Jean-surRichelieu et l'Île-aux-Noix. Au sujet de leurs activités, voir Jacques Castonguay, Les défis du fort Saint-Jean; l'invasion ratée des Américains en 1775 (Saint-Jean, Éditions du Richelieu, 1975), 132, 141-143; Réal Fortin, Bateaux et épaves du Richelieu (Saint-Jean, Éditions Mille Roches, 1977), 62-64, 81-85.

28 James Stewart Pritchard, «Ships, Men and Commerce in New France; A Study of Maritime Activity in New France», thèse de doctorat (Ph. D.), University of Toronto, $1971,10$. 
treinte, certaines peuvent franchir l'Atlantique à l'occasion ${ }^{29}$. Le ship ou navire-marchand, pour employer un néologisme, tient le second rang en importance parmi les bâtiments lancés au cours de cette période. Long-courrier adapté aux conditions de la navigation océanique, le navire-marchand provenant de la vallée laurentienne jauge entre 125 et 790 tonneaux, mais dans la très grande majorité des cas, il se situe entre 200 et 500 tonneaux. Le sloop enfin vient au troisième rang. De fort petite taille, ce voilier jauge habituellement entre 30 et 60 tonneaux, et il ne dépasse qu'exceptionnellement les 100 tonneaux. Construit exclusivement dans des chantiers maritimes établis sur les rives du fleuve entre Pointe-auPère et Montréal, le sloop semble destiné surtout au commerce fluvial et côtier, d'autant plus qu'il peut aisément travailler à l'échouage, tout comme certaines goélettes d'ailleurs.

En adoptant pour cadre de référence la taille et la morphologie des bâtiments lancés dans la vallée laurentienne entre 1760 et 1825 , il est possible de regrouper tous les chantiers maritimes identifiés au sein de trois ensembles spécifiques, chacun correspondant à une zone géographique particulière. Le premier ensemble, que nous avons nommé la zone estuarienne, comprend tous les chantiers établis dans les localités du golfe de même que celles situées sur les deux rives du fleuve, depuis la Gaspésie jusqu'à la pointe orientale de l'île d'Orléans. Le deuxième ensemble, baptisé zone méso-fluviale, englobe les chantiers implantés depuis la pointe orientale de l'île d'Orléans jusqu'à une ligne imaginaire coupant le fleuve entre Deschambault, sur la rive nord, et Lotbinière, sur la rive sud. Enfin, le dernier ensemble, dit zone fluviale, réunit tous les chantiers situés à l'ouest de cette ligne imaginaire. Sur le plan de la production des voiliers, chaque zone se comporte de façon distincte, même si à l'intérieur de chacune d'entre elles, les localités productrices de bâtiments ne sont pas toutes identiques.

Territorialement la plus vaste, la zone estuarienne regroupe une cinquantaine de centres de production, soit plus de la moitié de tous les chantiers maritimes établis dans la vallée du SaintLaurent. Cette zone se spécialise dans la construction de bâtiments de faible tonnage; ceux inférieurs à 100 tonneaux comptent pour 92,5 pour cent du total de sa production, et aucun bâtiment ne jauge plus de 300 tonneaux. La zone estuarienne se consacre essentiellement aussi à la mise en chantier de goélettes, celles-ci comp-

29 Par exemple, en 1797, une goélette construite à Québec, la Harbinger, mesurant 60 pieds de longueur et jaugeant 57 tonneaux, s'enregistre au port de Londres; en 1811, une autre de 58 tonneaux et d'une longueur de 52 pieds, construite à Montmagny, la Felicity, fait naufrage à Gibraltar. Ces cas ne sont pas isolés, et nul doute que ce type de bâtiment pouvait servir non seulement pour le cabotage mais aussi pour le commerce maritime. 


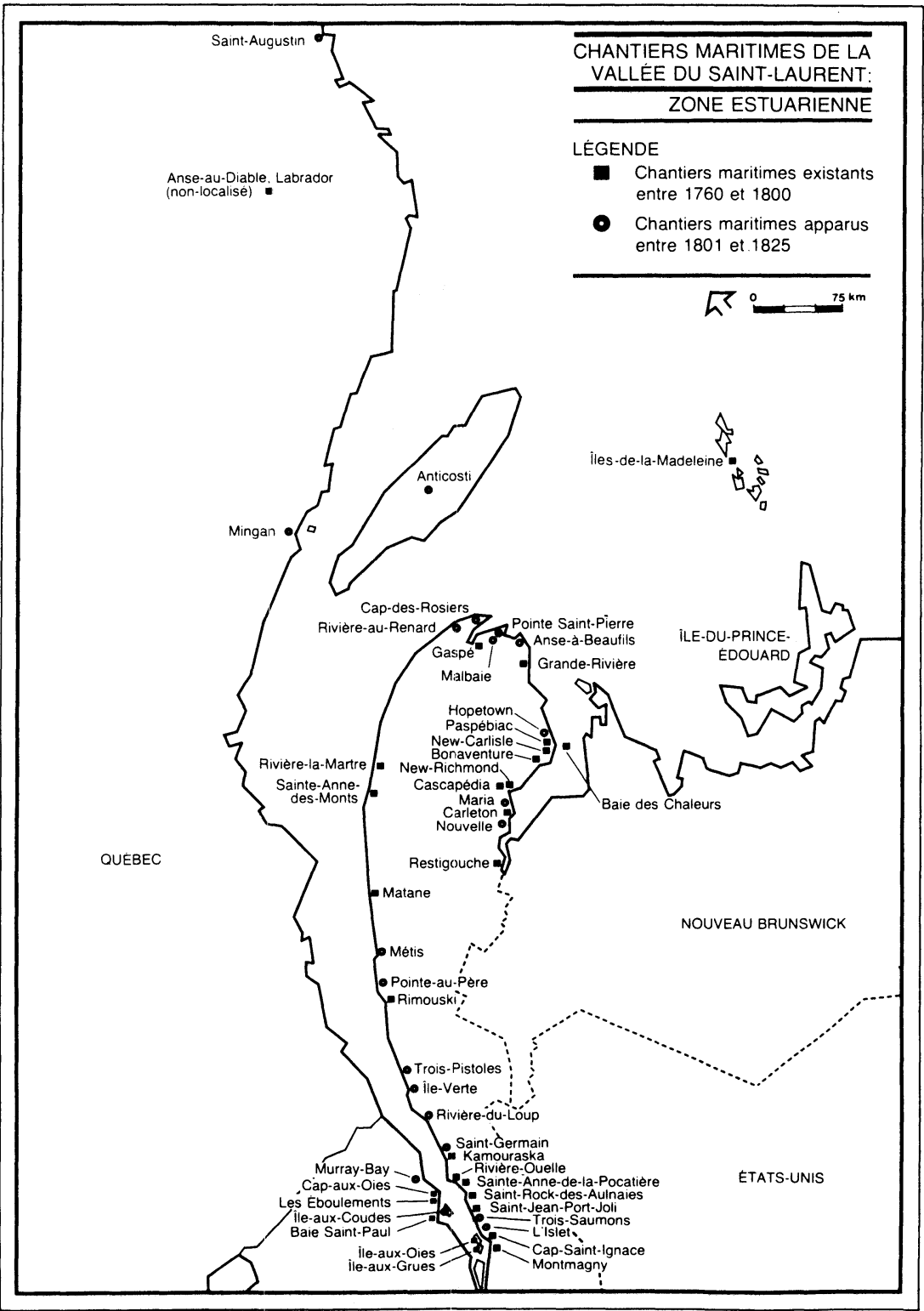




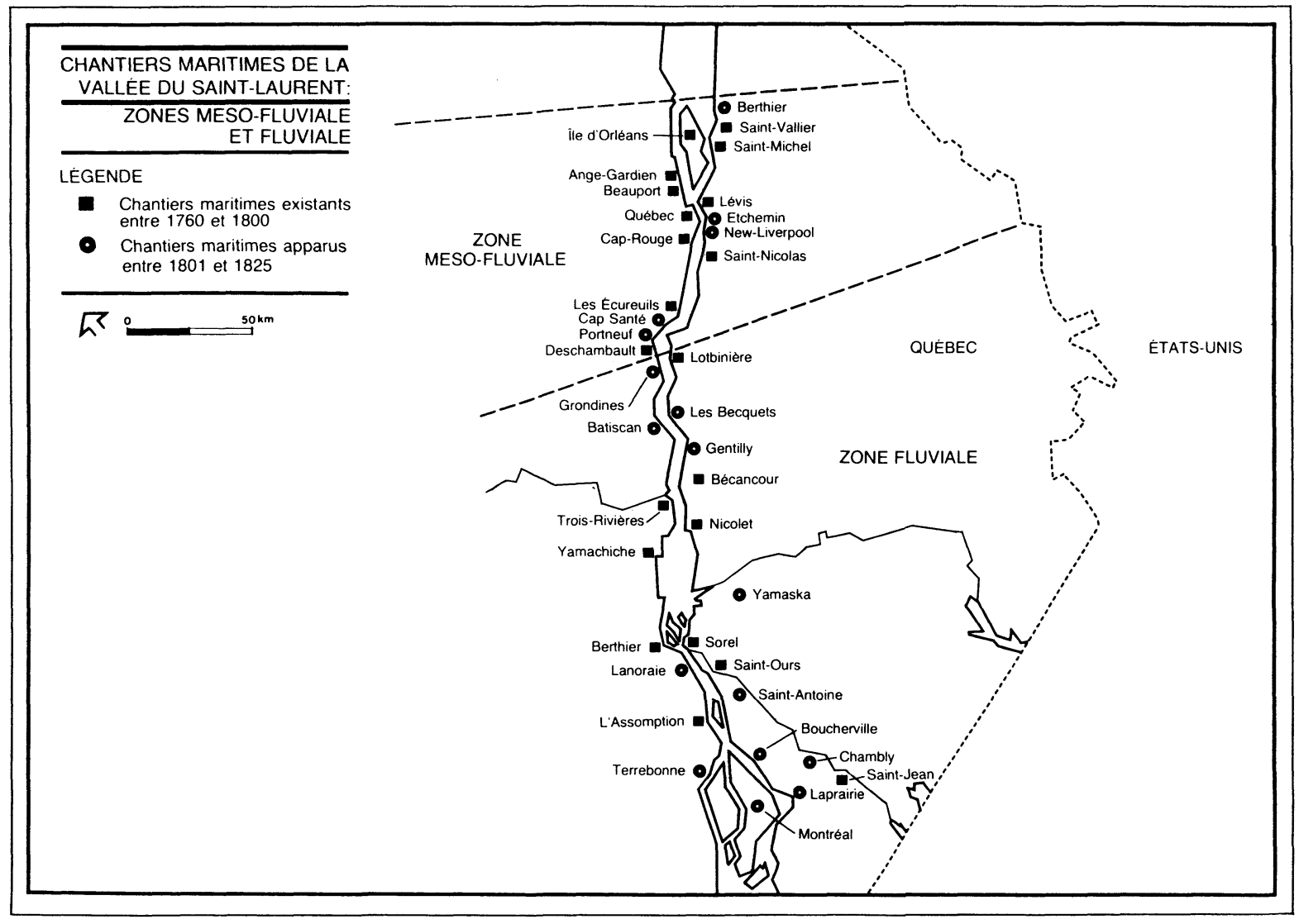


tant pour 82 pour cent de la production. Seulement 12 des 49 centres de production de cette zone lancent des bâtiments jaugeant plus de 100 tonneaux: les Îles-de-la-Madeleine, la région de la Baie des Chaleurs, New Richmond, New Carlisle, Paspébiac, Gaspé, Bonaventure, Malbaie, Matane, Saint-Jean-Port-Joli, l'Islet et Baie Saint-Paul. Les 37 autres centres se limitent à la mise en chantier de bâtiments inférieurs à 100 tonneaux: presque exclusivement des goélettes, quelques sloops et quelques chaloupes (à voile). Or, la construction de ce genre de bâtiments n'exige pas nécessairement d'installations permanentes; il suffit qu'une grève soit propice au lancement pour qu'on y entreprenne l'assemblage d'un voilier de faible tonnage ${ }^{30}$. Il y a donc de fortes chances pour que ces centres de production ne soient que des chantiers maritimes temporaires, surtout si l'on considère le rythme très sporadique des mises à l'eau de bâtiments dans la plupart de ces endroits. En fait, si l'on tient compte de la taille des bâtiments, de la diversité des types de navires construits en un même lieu et de la régularité des lancements sur l'ensemble de la période, quelques centres de production seulement émergent au sein de la zone estuarienne: la région de la Baie des Chaleurs, et plus particulièrement Paspébiac, ainsi que Gaspé et Baie Saint-Paul. En outre, bien qu'ils se consacrent principalement à la construction de goélettes, quatre autres endroits se distinguent aussi par la fréquence de lancement de bâtiments: Îles-de-la-Madeleine, Carleton, Saint-Jean-Port-Joli et Montmagny. Partout ailleurs, la construction navale semble une activité plutôt ponctuelle.

Les chantiers maritimes de la zone méso-fluviale se distinguent singulièrement de ceux de la zone estuarienne. Géographiquement la plus restreinte, la zone méso-fluviale ne rassemble que 16 centres de production. Mais 70,5 pour cent des bâtiments sortis de ces chantiers jaugent plus de 100 tonneaux. De plus, la catégorie de bâtiments la plus répandue dans cette zone est celle du naviremarchand, qui compte pour le tiers de sa production, la goélette venant au deuxième rang avec 25,7 pour cent. D'ailleurs, la goélette provenant de la zone méso-fluviale est généralement de dimensions plus grandes que celle de la zone estuarienne: sa jauge oscille, en effet, entre 50 et 150 tonneaux plutôt qu'entre 30 et 100. La production des chantiers de la zone méso-fluviale affiche aussi une plus grande variété au niveau des différents types de bâtiments construits; en plus de produire les mêmes voiliers que ceux de la zone estuarienne, les chantiers de la zone méso-fluviale lancent aussi des barques, quelques bateaux et vapeurs, bâtiments probablement destinés surtout au commerce fluvial et côtier. Même si

$30 \quad$ Michel Desgagnés, Les goélettes de Charlevoix (Montréal, Leméac, 1977), 53. 


\section{TABLEAU 2}

Nombre minimal de bâtiments construits dans la vallée du Saint-Laurent, 1760-1825 (classement par types)

\begin{tabular}{|c|c|c|c|c|}
\hline Types & $\begin{array}{c}\text { Zone } \\
\text { estuarienne }\end{array}$ & $\begin{array}{l}\text { Zone } \\
\text { méso- } \\
\text { fluviale }\end{array}$ & $\begin{array}{c}\text { Zone } \\
\text { fluviale }\end{array}$ & Total \\
\hline Barque (bark) & - & 31 & 2 & 33 \\
\hline Bateau (batteau) & - & 1 & 2 & 3 \\
\hline Brick (brig) & 9 & 35 & 10 & 54 \\
\hline Brigantin (brigantine) & 12 & 33 & 7 & 52 \\
\hline Chaloupe (shallop) & 18 & 4 & 1 & 23 \\
\hline Goélette (schooner) & 338 & 94 & 61 & 493 \\
\hline Navire-Marchand (ship) & 11 & 121 & 34 & 166 \\
\hline Seneau (snow) & 3 & 20 & 19 & 42 \\
\hline Sloop (sloop) & 21 & 25 & 33 & 79 \\
\hline \multirow[t]{2}{*}{ Vapeur (steam vessel) } & - & 3 & 15 & 18 \\
\hline & 412 & 367 & 184 & 963 \\
\hline
\end{tabular}

\section{TABLEAU 3}

Nombre minimal de bâtiments construits dans la vallée du Saint-Laurent, 1760-1825 (classement par jauge)

Jauge

(en tonneaux)

0 à 49

50 à 99

100 à 199

200 à 299

300 à 399

400 à 499

500 à 599

600 à 999

1000 et plus
Zone
estuarienne

259

121

25

7

$-$

$-10$

$-$

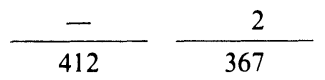

mésofluviale

61

47

67
37
Zone

fluviale

34

51

354

219

133

103

89

31

22

46

9

11

6

46

elle ne contient que 16 centres de production comparativement aux 49 de la zone estuarienne, la zone méso-fluviale en compte autant, en chiffres absolus, qui construisent des voiliers jaugeant plus de 100 tonneaux: Ange-Gardien, Beauport, île d'Orléans, Lévis, Québec, New Liverpool, Saint-Nicolas, Cap-Rouge, Cap-Santé, Portneuf et Deschambault. Parmi ces localités, les chantiers de l'île 
d'Orléans, Québec, Portneuf et Deschambault dominent, tant sur le plan de la taille et de la diversité des bâtiments construits que sur celui de leur cadence de production.

La zone fluviale, enfin, englobe 22 centres de production et elle possède des caractéristiques qui l'apparentent tantôt à l'une, tantôt à l'autre des deux zones décrites précédemment. Tout comme la zone méso-fluviale, la zone fluviale produit davantage de bâtiments de grandes dimensions, ceux-ci constituant 53,8 pour cent de sa production totale. La goélette y tient, par contre, la première place, comme pour la zone estuarienne, mais elle s'y avère proportionnellement moins importante pour n'atteindre que 33,15 pour cent des divers types de bâtiments construits. La goélette mise en chantier dans la zone fluviale ressemble d'ailleurs davantage, par sa taille, à celle de la zone méso-fluviale. La construction du navire-marchand occupe une place relativement importante dans la production de la zone fluviale $(18,48$ pour cent) et ses dimensions sont identiques à celles du même type de bâtiments lancés dans la zone méso-fluviale. Compte tenu, par ailleurs, de la proportion élevée du nombre de goélettes et de sloops dans la production de la zone fluviale, celle-ci ressemble davantage à la zone estuarienne; mais considérée sous l'angle de la diversité des types de bâtiments produits, elle s'apparente à nouveau à la zone mésofluviale. En fait, la construction du bateau à vapeur semble en voie de devenir la marque distinctive de la zone fluviale, bien qu'elle n'ait été entreprise qu'une quinzaine d'années avant la fin de la période à l'étude. La plupart des centres de production situés dans cette zone fabriquent des bâtiments de faible tonnage, mais TroisRivières, Bécancour, Terrebonne, Sorel, Laprairie, Montréal, Saint-Ours et Chambly lancent aussi des bâtiments supérieurs à 100 tonneaux. Les chantiers de Sorel et de Montréal dominent nettement tous les autres par la diversité des types de bâtiments construits et par la régularité des lancements, ceux des villages de Bécancour et Saint-Ours apparaissant comme des pôles secondaires.

En résumé, la construction navale se pratique dans plus de 80 localités de la vallée laurentienne entre 1760 et 1825 . Au total, une dizaine de classes différentes de bâtiments sont produits, les plus courants étant la goélette, le navire-marchand et le sloop. On note encore un certain degré de spécialisation régionale des divers chantiers maritimes, compte tenu de la taille et des classes de bâtiments construits. Enfin, la construction navale semble revêtir une plus grande importance pour un petit nombre seulement de localités au sein de chaque zone de production. 


\section{4- La construction navale à Québec, 1760-1825}

Si la mise en chantier de bâtiments s'effectue dans la plupart des localités qui s'échelonnent le long du fleuve, la construction navale n'a certainement pas la même incidence ni le même effet d'entraînement partout; et son envergure, à un endroit donné, nous semble tributaire du type de marché auquel elle s'articule.

Bon nombre de chantiers maritimes, en effet, répondent uniquement à des besoins locaux comme, par exemple, ceux établis aux Îles-de-la-Madeleine. Tous les bâtiments construits à cet endroit durant le premier quart du XIX ${ }^{\mathrm{e}}$ siècle appartiennent à des marchands ou des navigateurs madelinots. Quelques-uns seulement, après avoir navigué pendant un certain nombre d'années, sont «exportés», c'est-à-dire vendus à des propriétaires résidant ailleurs: deux bâtiments sont ainsi acheminés vers Québec, deux autres vers l'Angleterre et un dernier vers Halifax. Au cours de la même période, la construction navale à Deschambault répond essentiellement aussi aux besoins de navigateurs et de marchands de cette localité, tout comme celle pratiquée à Montmagny, même si, à ce dernier endroit, quelques bâtiments sont parfois destinés à des résidents de paroisses voisines telles Berthier-en-bas, L'Islet et Cap-Santé.

Cette orientation de la construction navale à des fins locales ou tout au plus régionales s'étend à la très grande majorité des chantiers qui ont pu être identifiés. En fait, un nombre passablement limité de chantiers maritimes écoulent une partie appréciable de leur production ailleurs que sur le seul marché domestique: ceux de Montréal, Sorel, Portneuf, Québec, Baie Saint-Paul, Gaspé, Baie des Chaleurs et Paspébiac. Durant le premier quart du XIX ${ }^{e}$ siècle, ces huit endroits concentrent d'ailleurs 79,63 pour cent du tonnage global produit par l'ensemble des chantiers maritimes laurentiens. Les chantiers de Québec affichent une nette primauté, pour fournir à eux seuls plus de la moitié du tonnage global, soit 52,39 pour cent. Les chantiers montréalais se classent au deuxième rang avec 16,8 pour cent, suivis par ceux de Sorel avec 2,78 pour cent, les autres s'équivalant entre eux, chacun produisant un peu moins de deux pour cent.

Les chantiers maritimes de Québec manifestent les mêmes tendances de production que l'ensemble de la zone méso-fluviale, et pour cause, puisque des 367 bâtiments construits dans cette zone entre 1760 et 1825, 288 sortent des chantiers de Québec. Ces derniers exercent un monopole quasi absolu sur la construction des bâtiments de fort tonnage, produisant 235 des 259 navires de plus de 100 tonneaux mis en chantier dans la zone méso-fluviale. Les 
chantiers québécois sont aussi les plus diversifiés de la zone, lançant tous les types de bâtiment à l'exception de la chaloupe; ils produisent tous les seneaux, bateaux et barques mis à l'eau dans cette zone au cours de la période, et 109 des 121 naviresmarchands.

La prépondérance de Québec dans la construction navale non seulement de la zone méso-fluviale, mais encore de l'ensemble de la vallée du Saint-Laurent, explique sans doute pourquoi leurs courbes de production respectives adoptent en général un comportement semblable.

Mais en $1801,1806,1814,1817$ et 1821 , les chantiers maritimes de la vallée laurentienne connaissent de légères hausses de production tandis que ceux de Québec stagnent ou enregistrent des baisses. Inversement, en 1816 et 1819, ce sont ceux de Québec qui affichent des augmentations, alors que, dans la vallée du SaintLaurent, la construction navale marque le pas ou accuse un léger recul. Ces écarts semblent imputables au fait que les chantiers maritimes de Québec ne répondent que partiellement aux mêmes stimuli que les autres chantiers; la majeure partie de leur production est destinée au marché extérieur, alors que pour les autres chantiers, le marché domestique demeure habituellement le principal débouché.

Que la plupart des navires construits à Québec soient vendus à l'extérieur n'étonnera évidemment personne, les historiens ayant déjà souligné l'étroite dépendance de la construction navale québécoise envers le marché britannique ${ }^{31}$. Mais, grâce aux certificats d'enregistrement de navires, il est désormais possible d'affiner nos connaissances sur ce marché britannique qui a été présenté jusqu'ici de façon monolithique, d'en supputer l'importance relative pour les chantiers de Québec par rapport au marché domestique, et d'identifier les principaux foyers d'importation, qu'ils soient situés en Grande-Bretagne ou ailleurs.

La première mention d'exportation d'un bâtiment construit à Québec remonte à l'année même de l'ouverture des registres de certificats, soit en 1787, ce qui n'exclut pas la possibilité qu'il y en ait eu auparavant. Cette année-là, huit marchands écossais de la région de Glasgow se portent acquéreurs, par l'entremise d'un fondé de pouvoir de passage à Québec, le capitaine James Farric, du nouveau voilier Alfred, un trois mâts d'une longueur de 77 pieds jaugeant 205 tonneaux. Rapatrié aussitôt, le certificat d'enregistrement du Alfred est transféré au port de Greenock, situé

31 En particulier Faucher, op. cit., 232, et Ouellet, Le Bas-Canada, 249. 
PRODUCTION (tonnage annuel) DES CHANTIERS NAVALS DE LA VALLÉE DU SAINT-LAURENT, 1800-1825

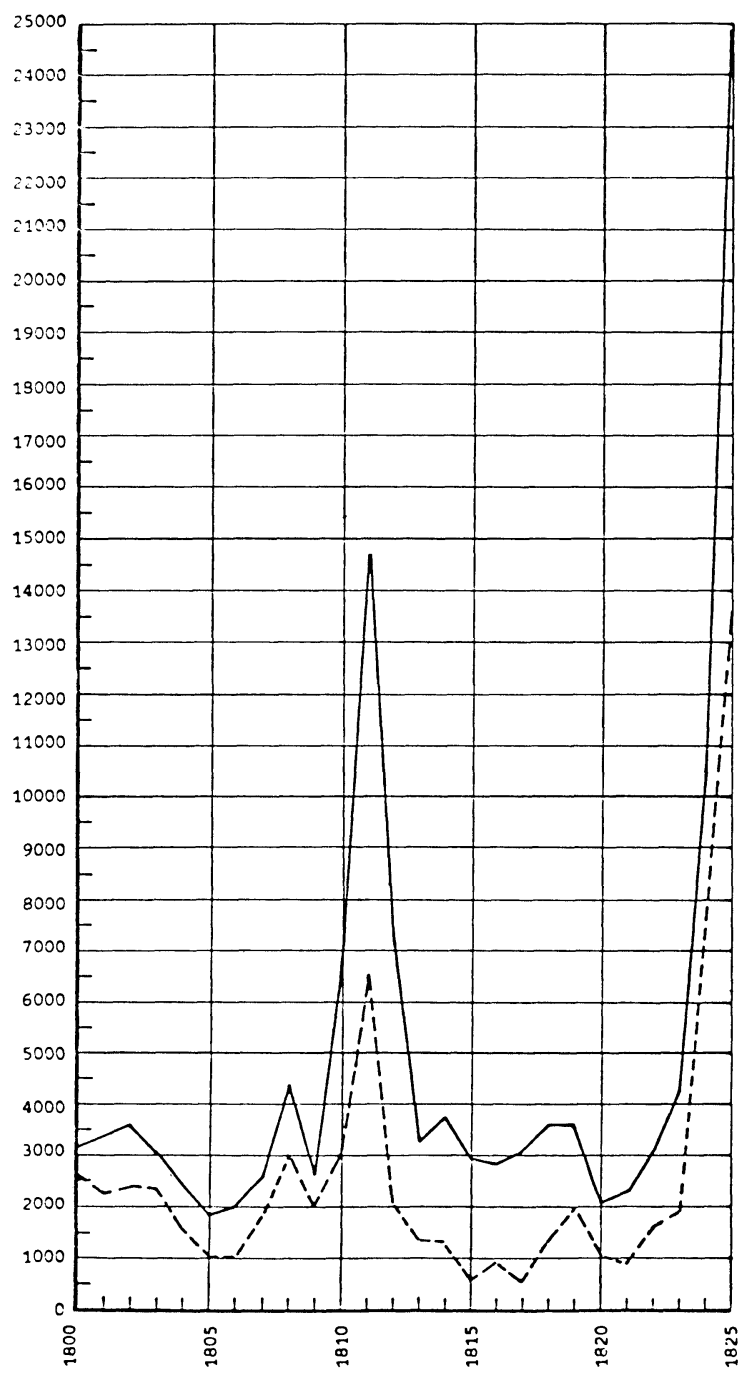

LÉGENDE:

Vallée du Saint-Laurent

Ville de Québec - - - 
tout près de Glasgow. À compter de cette date et jusqu'en 1824 inclusivement, les chantiers de Québec lancent 234 bâtiments totalisant 55660 tonneaux. Une part relativement faible de cette production est absorbée par le marché local puisque 65 pour cent du tonnage global est exporté sur le marché nord-atlantique, soit vers les îles britanniques, soit vers d'autres colonies. En réalité, les colonies britanniques constituent un marché négligeable. Si les Antilles revêtent une certaine importance pour les exportateurs de bois de Québec, il n'en va pas de même pour les constructeurs de navires; durant toute la période, une seule goélette jaugeant 122 tonneaux, la Saint Lawrence, est exportée en Jamaïque, en 1819. Les colonies de Terre-Neuve et de Nouvelle-Écosse n'offrent guère plus de débouchés, n'achetant que cinq petits voiliers en 28 ans, pour un tonnage total de 409 tonneaux ${ }^{32}$. En somme, les îles britanniques constituent pratiquement la seule clientèle "étrangère» des chantiers navals de la ville de Québec.

La clientèle britannique n'est cependant pas aussi homogène qu'on pourrait être porté à le croire. D'abord, elle n'occupe pas tout le territoire de Grande-Bretagne, mais seulement quelques endroits bien déterminés. Ensuite, ses besoins en bâtiments de mer fluctuent dans le temps, variations qui semblent jusqu'à un certain point du moins, reliées à sa localisation géographique.

De 1787 à 1824, l'Angleterre absorbe 73,4 pour cent de tous les bâtiments québécois exportés vers la Grande-Bretagne; l'Écosse pour sa part en achète 20,4 pour cent, tandis que l'Irlande n'en acquiert que 2,9 pour cent. En Angleterre, les principaux acheteurs de voiliers québécois se concentrent à Londres ou dans sa région; les trois-quarts du tonnage importé en effet par l'Angleterre y sont acheminés. Le seul autre foyer d'importation de quelque importance se situe sur la côte ouest, à Liverpool. Mais les besoins des armateurs de Liverpool ne correspondent pas à ceux de la région londonienne, car ils importent des bâtiments d'un tonnage comparativement plus faible; tous les voiliers qui s'y dirigent jaugent entre 100 et 300 tonneaux, à quelques rares exceptions près, tandis que ceux acquis par les Londoniens oscillent souvent entre 300 et 500 tonneaux. La demande pour des bâtiments québécois par des armateurs d'autres régions d'Angleterre, demeure très faible et très irrégulière au cours de la période, ce qui laisse entrevoir qu'elle ne dépend pas des mêmes facteurs. Par exemple, l'exportation de bâtiments vers les ports de New Castle, Sunderland et Hull, tous

32 Terre-Neuve et la Nouvelle-Écosse achètent par contre plusieurs bâtiments construits dans les chantiers de la zone estuarienne. 


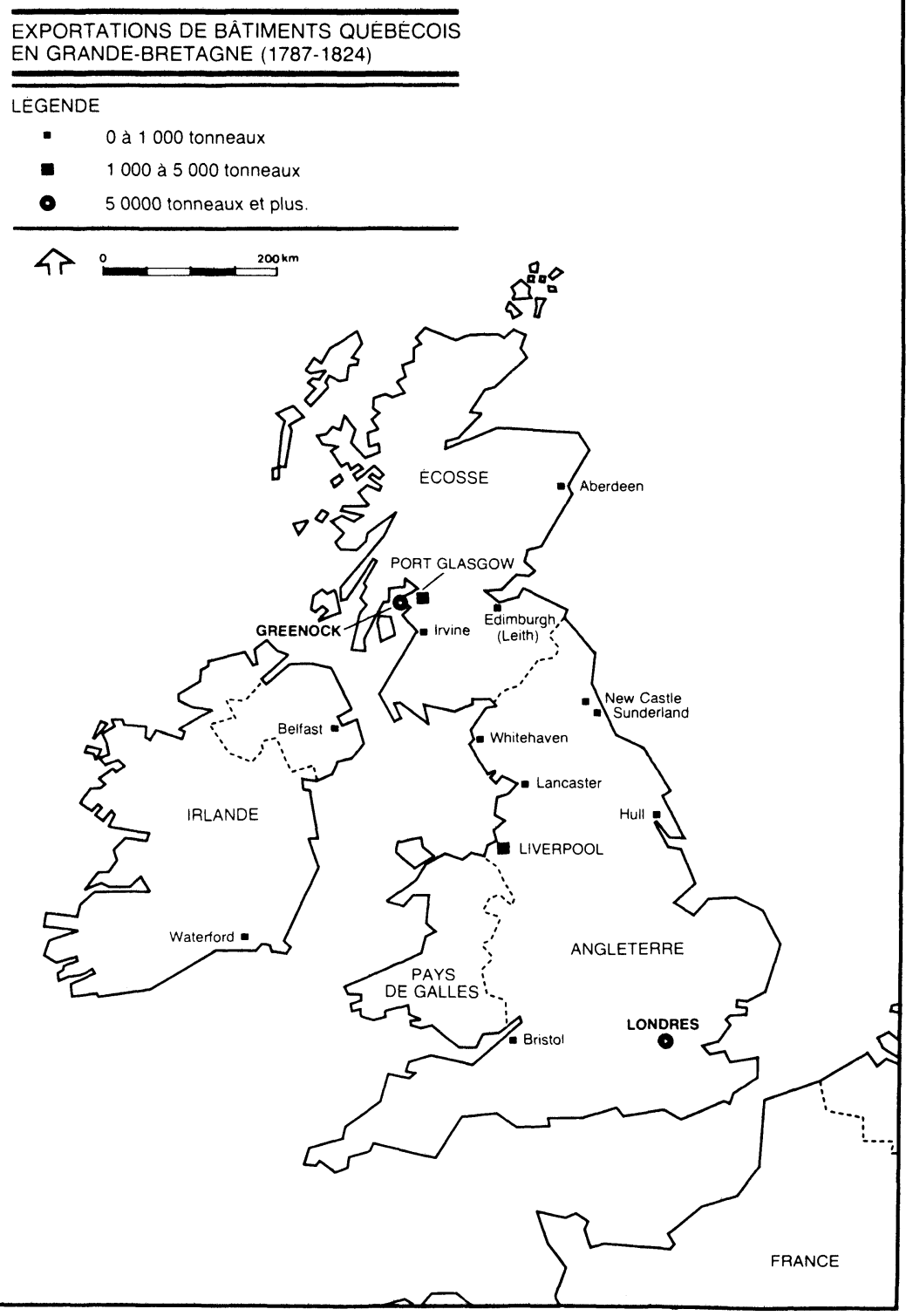


situés sur la côte est de l'Angleterre, s'inscrit entre les années 1809 et 1815 , ce qui s'explique sans doute par la conjoncture politique européenne à ce moment-là. Par contre, les exportations de bâtiments vers Bristol et Whitehaven, situés sur la côte ouest, sont trop peu fréquentes et trop dispersées dans le temps pour permettre d'établir des corrélations.

En Écosse, la clientèle est géographiquement plus concentrée encore qu'elle ne l'est en Angleterre, puisque 87,5 pour cent du tonnage importé est dirigé vers Greenock et Port Glasgow, situés à quelques kilomètres seulement l'un de l'autre. Là aussi, les achats de bâtiments effectués par des armateurs d'autres régions semblent liés à des facteurs ponctuels. Par exemple, Aberdeen, situé sur la côte est de l'Écosse, adopte le même comportement que les ports anglais de New Castle, Sunderland et Hull, pour n'importer des bâtiments québécois qu'entre 1809 et 1811. Enfin, les quelques voiliers québécois acheminés vers les ports irlandais de Belfast et de Waterford au cours de la même période sont trop peu nombreux pour permettre d'évaluer l'importance relative de ces deux foyers d'importation. En fait, le marché irlandais apparaît seulement à la fin des années 1810 . Ce n'est qu'en 1819 qu'un bâtiment construit à Québec, le Olive Branch, un brick de 206 tonneaux, est expédié pour la première fois vers Belfast. Par contre, au même moment, les exportations vers l'Écosse marquent un temps d'arrêt, avec l'envoi vers Greenock en 1820, du brick Betsey, jaugeant 211 tonneaux. Seule la poursuite de la recherche sur la période subséquente permettra de savoir s'il ne s'agit que d'une coïncidence ou s'il faut voir dans ce phénomène le début d'un réaménagement des foyers d'importation de bâtiments québécois au sein des îles britanniques.

\section{Conclusion}

Ce bref aperçu sur la construction navale à Québec entre 1760 et 1825 soulève certainement autant de questions qu'il n'apporte d'éclaircissements. Rappelons qu'il s'agit d'abord et avant tout d'un bilan partiel, d'autres corrélations que celles illustrées ici pouvant être établies au moyen de la même source. Par exemple, les historiens relient souvent les débuts de la construction navale à Québec au blocus continental imposé par la France au cours de la première décennie du XIX ${ }^{\mathrm{e}}$ siècle; l'analyse des certificats d'enregistrement de navires laisse plutôt entrevoir que cet événement n'est venu qu'accélérer un processus dont le démarrage, amorcé plus tôt, dépendrait d'autres facteurs.

Dans un autre ordre d'idées, il est possible aussi d'identifier avec passablement de justesse les intérêts financiers qui, en dernier 
ressort, sous-tendent la construction navale à Québec au cours de cette période. Ainsi, les principaux constructeurs du tournant du siècle, les Munn, Beatson, Black et King sont non seulement originaires d'Écosse, mais ils ont partie liée avec des marchands ou des firmes de la région Greenock-Glasgow. Bien plus, leurs partenaires commerciaux établis à Québec sont habituellement de même origine et ils portent souvent des patronymes identiques à ceux d'autres associés oeuvrant en Écosse.

Les certificats d'enregistrement peuvent encore fournir des informations intéressantes sur le plan technique. Par exemple, l'absence fréquente d'ornementation comme les bouteilles ou les figures de proue incite à croire que les bâtiments construits à Québec entre 1760 et 1825 devaient afficher une apparence relativement rustique.

Rappelons, par ailleurs que ce bilan revêt un caractère tout aussi provisoire que partie. La poursuite des recherches sur les années postérieures à 1825 pourrait nous amener à remettre en question la pertinence et l'utilité de certains concepts surgis à la suite du traitement préliminaire des données accumulées au cours de la première phase des travaux, comme celui des zones de production. Elle risque par contre de rendre significatives et compréhensibles d'autres données engrangées pendant la première phase, mais qui n'ont pas été signalées dans le présent article. C'est le cas notamment des renseignements recueillis à propos des chantiers maritimes montréalais. À compter de 1805, Montréal exporte proportionnellement à sa production totale, presque autant de bâtiments que Québec, et ce, vers les mêmes points de vente. Les chantiers montréalais semblent donc, à première vue, concurrencer ceux de Québec sur le marché des îles britanniques. Il peut cependant ne s'agir que d'un phénomène de complémentarité, les intérêts financiers impliqués dans la construction navale à Montréal étant souvent intimement liés à ceux insufflant cette activité à Québec.

Tout partiel et provisoire qu'il soit, cet aperçu démontre que l'histoire de la construction navale à Québec au XIX ${ }^{\mathrm{e}}$ siècle mérite d'être révisée en profondeur. 